\title{
Caracterização física e química das frações do fruto atemoia Gefner
}

\author{
Physical and chemical characterization of fractions of fruit atemoya Gefner
}

\author{
Luciana Soares da Cruz ${ }^{I^{*}}$ Rafaela Zambaldi Lima $^{\mathrm{I}}$ Celeste Maria Patto de Abreu \\ Angelita Duarte Corrêa ${ }^{I}$ Luciana de Matos Alves Pinto ${ }^{I}$
}

\section{RESUMO}

A atemoia é um fruto híbrido derivado do cruzamento entre a fruta-do-conde, mais conhecida como ata (Annona squamosa L.), com a cherimoia (Annona cherimola Mill.). Este trabalho foi realizado com o objetivo de caracterizar os constituintes químicos das frações casca, polpa e semente de atemoia Gefner. Os frutos adquiridos foram selecionados, pesados e medidos. Foram separados em casca, polpa, semente e eixo floral, que também foram pesados para determinar suas proporções. Para o restante dos frutos, foram separadas as frações em sete repetições de 13 frutos. Em seguida, as frações casca, polpa e sementes foram liofilizadas e armazenadas em freezer. Foi determinada a proporção das frações do fruto, a composição centesimal, o ácido ascórbico, os açúcares totais, os minerais e alguns compostos bioativos. A polpa representou cerca de $60 \%$ do peso do fruto, enquanto a casca $28,13 \%$ e as sementes 8,34\%. O fruto apresentou, em média, 56 sementes, com diâmetro longitudinal de $10,79 \mathrm{~cm}$ e diâmetro transversal de $26,64 \mathrm{~cm}$. Os maiores teores de proteína bruta, extrato etéreo e fibra alimentar foram encontrados nas sementes e casca. A casca se destacou nos teores de cinzas. A polpa apresentou os maiores teores de ácido ascórbico e açúcares totais em relação às outras frações. A ordem da composição de macronutrientes na casca, na polpa e nas sementes da atemoia foi $\mathrm{K}>\mathrm{P}>\mathrm{Ca}>\mathrm{Mg}$; para os micronutrientes na casca e nas sementes, foi $\mathrm{Fe}>\mathrm{Zn}>\mathrm{Cu}>\mathrm{Mn}>\mathrm{S}$ e, na polpa, foi $\mathrm{Fe}>\mathrm{Zn}>\mathrm{Mn}>\mathrm{Cu}>\mathrm{S}$. A casca apresentou os níveis mais altos de inibidores de tripsina e atividade hemaglutinante. Os teores de compostos fenólicos foram relativamente baixos no fruto.

Palvras-chave: Annona, composição química, nutrientes, compostos bioativos.

\section{ABSTRACT}

The atemoya is a hybrid fruit derived from the cross between the sugar apple (fruta-do-conde), better known as ata (Annona squamosa L.) with the cherimoya (Annona cherimola
Mill.). The aim of this research was to characterize the chemical constituents of the fractions skin, pulp and seed of the hybrid fruit atemoya, variety Gefner. The fruits purchased and selected were weighed and measured. They were separated into skin, pulp, seed and floral axis which were also weighted to determine their proportions. For the rest of the fruits, the fractions were separated into 7 replicates of 13 fruits. Then, the fractions skin, pulp and seeds were freeze-dried and stored in freezer. The proportion of the fruit fractions, the centesimal composition, ascorbic acid, total sugars, minerals and some bioactive compounds were determined. The pulp represented about $60 \%$ of the fruit weight, while the skin $28.13 \%$ and the seeds $8.34 \%$. The fruit presented on the average 56 seeds, with longitudinal diameter of $10.79 \mathrm{~cm}$ and transversal diameter of $26.64 \mathrm{~cm}$. The largest contents of crude protein, ether extract and dietary fiber were found in both seeds and skin. The skin stood out in the ash contents. The pulp showed the highest contents of ascorbic acid and total sugars in relation to the other fractions. The order of the composition of macronutrients in the skin, pulp and seeds of the atemoya was $K>P>C a>M g$; for the skin and seed micronutrients were $\mathrm{Fe}>\mathrm{Zn}>\mathrm{Cu}>\mathrm{Mn}>\mathrm{S}$ and in the pulp were $\mathrm{Fe}>\mathrm{Zn}>\mathrm{Mn}>\mathrm{Cu}>\mathrm{S}$. The skin presented the highest levels of trypsin inhibitors and hemagglutinating activity. The phenolic compounds were relatively low in the fruit.

Key words: Annona, chemical composition, nutrients, bioactive compounds.

\section{INTRODUÇÃO}

As frutas são boas fontes de nutrientes, porém os subprodutos como a casca e as sementes de vegetais costumam ser descartadas pela indústria e consumidores. Esses subprodutos poderiam ser aproveitados como fonte alternativa de nutriente

\footnotetext{
'Departamento de Química, Universidade Federal de Lavras (UFLA), 37200-000, Lavras, MG, Brasil. E-mail: lucianasoaresdacruz@yahoo.com.br. *Autor para correspondência. 
e, serem utilizados com segurança na alimentação humana.

A atemoia é um híbrido derivado do cruzamento entre um fruto tropical, a fruta-do-conde, mais conhecida como ata (Annona squamosa L.), com a cherimoia (Annona cherimola Mill.), nativa das regiões andinas do Chile, Peru, Bolívia, Equador e em locais de clima ameno. Cerca de mil hectares de atemoia são plantados no Brasil (CAXITO, 2009).

A atemoia pode reunir características desejáveis e relevantes, oriundas das duas espécies que participaram do seu cruzamento, sendo importante determinar seus constituintes químicos. Devido aos poucos artigos encontrados na literatura sobre a atemoia, o objetivo deste trabalho foi caracterizar física e quimicamente as frações da casca, polpa e sementes de atemoia Gefner.

\section{MATERIAL E MÉTODOS}

O pomar onde a atemoia foi cultivada está situado no município de Jaíba, localizado no norte de Minas Gerais, durante o ciclo agrícola 2009/2010. Os frutos foram colhidos no estágio de vez, acondicionados em caixas de papelão e transportados para o Laboratório de Bioquímica no município de Lavras-MG. Posteriormente, as atemoias foram abertas e as frações casca, polpa, sementes e eixo floral foram separadas, pesadas e as sementes foram contabilizadas. As partes dos frutos foram liofilizadas, moídas em moinho e armazenadas em freezer até a realização das análises. Foi determinado o peso dos frutos em balança semianalítica com sensibilidade de $0,001 \mathrm{~g}$. O diâmetro longitudinal e transversal dos frutos foi mensurado com o uso de um paquímetro digital.

Para determinar a umidade, as amostras das frações do fruto, casca, polpa e sementes foram secas em estufa, a $65^{\circ} \mathrm{C}$, até atingirem peso constante (AOAC, 2005). A proteína bruta ( $\mathrm{N}$ x 6,25), o extrato etéreo, as cinzas, as fibras alimentares e o extrato não nitrogenado $[\mathrm{ENN}=100$ - (proteína bruta + extrato etéreo + cinzas + fibra alimentar)] foram realizados segundo a metodologia descrita pela AOAC (2005).

$\mathrm{O}$ ácido ascórbico foi determinado utilizando o método de Tillman, sendo extraído com ácido oxálico e, após filtração, dosado no extrato, utilizando o ácido ascórbico como padrão (STROHECKER \& HENNING, 1967). Os resultados foram expressos em $\mathrm{mg} 100 \mathrm{~g}^{-1}$.

Os açúcares totais foram extraídos pelo método de Lane-Enyon, segundo a AOAC (2005), determinados pelo método de Antrona (DISCHE, 1962) e expressos em g $100 \mathrm{~g}^{-1}$.
Os teores dos macrominerais ( $\mathrm{P}, \mathrm{K}, \mathrm{Ca}, \mathrm{Mg}$ e $\mathrm{S})$ e dos microminerais $(\mathrm{Cu}, \mathrm{Mn}, \mathrm{Zn}$ e $\mathrm{Fe}$ ) foram determinados segundo MALAVOLTA et al. (1997), expressos em $\mathrm{g} 100 \mathrm{~g}^{-1}$ e $\mathrm{mg} \mathrm{kg}^{-1}$, respectivamente. $\mathrm{O}$ fósforo e o enxofre foram determinados por colorimetria; ferro, zinco, manganês, cobre, magnésio e cálcio por espectrofotometria de absorção atômica e potássio por fotometria de chama.

Os inibidores de tripsina foram determinados segundo o método de KAKADE, et al. (1969), expressos em $\mu \mathrm{mol} \mathrm{min}^{-1} \mathrm{~g}^{-1} \mathrm{MS}$, utilizando BAPNA (benzoil-DL-arginina-p-nitro-anilida) como substrato.

A extração dos compostos fenólicos foi realizada com metanol $50 \%$, em refluxo, por três vezes consecutivas a $80^{\circ} \mathrm{C}$ e os extratos reunidos, evaporados até $25 \mathrm{~mL}$ e submetidos à dosagem de compostos fenólicos, utilizando-se o reagente de Folin-Denis, expressos em mg 100g-1 (AOAC, 2005).

As lectinas foram extraídas com solução salina $\left(\mathrm{NaCl} 0,85 \mathrm{~g} 100 \mathrm{~mL}^{-1}\right)$ por três horas. Foi utilizada uma placa de microtitulação, na qual foram adicionadas diluições na base 2 do extrato e, em seguida, foi adicionada uma suspensão de eritrócitos a $2 \%$ de sangue humano tipo $\mathrm{A} \mathrm{Rh}+$. Após uma hora, determinou-se qual a maior diluição capaz de promover hemaglutinação (UH mg proteína ${ }^{-1}$ ) (CALDERÓN DE LA BARCA et al., 1985).

$\mathrm{O}$ experimento foi conduzido em delineamento inteiramente casualizado em esquema fatorial $3 \times 7$, sendo tratamentos (casca, polpa e sementes) e sete repetições, totalizando 91 frutos.

As análises estatísticas foram realizadas segundo técnicas usuais do software Sisvar. Quando a análise de variância mostrou diferença significativa, o teste de Tukey foi usado para comparação das médias, com probabilidade de $5 \%$.

\section{RESULTADOS E DISCUSSÃO}

Proporção das frações, diâmetro longitudinal e transversal e número de sementes

Observa-se que a polpa constituiu cerca de $60 \%$ do peso do fruto, enquanto que a casca e sementes $28,13 \%$ e $8,34 \%$, respectivamente, representando $36 \%$ do total. O eixo floral constituiu de $1,4 \%$ do peso. A atemoia apresentou, em média, 56,0 $0 \pm 9,0$ sementes, diâmetro longitudinal de $10,79 \pm 1,23 \mathrm{~cm} \mathrm{e}$ diâmetro transversal de $26,64 \pm 1,7 \mathrm{~cm}$ (Tabela 1).

Os resultados deste trabalho são semelhantes aos encontrados por NEVES \& YUHARA (2003), que, caracterizando os frutos de atemoia, Gefner, produzidos no norte do Paraná, 
Tabela 1 - Peso das frações de atemoia Gefner e respectivas proporções.

\begin{tabular}{lll}
\hline Amostra & \multicolumn{1}{c}{$(\mathrm{g})$} & $\mathbf{( \% )}$ \\
\hline Fruto & $321,09 \pm 28,60$ & 100 \\
Casca & $90,19 \pm 9,65$ & $28,13 \pm 2,31$ \\
Polpa & $193,58 \pm 20,46$ & $60,26 \pm 3,05$ \\
Sementes & $26,79 \pm 4,68$ & $8,34 \pm 1,23$ \\
Eixo floral & $4,38 \pm 0,50$ & $1,36 \pm 0,12$ \\
Perdas & $6,52 \pm 3,72$ & $2,03 \pm 1,2$ \\
\hline
\end{tabular}

*Os dados são a média de 7 frutos \pm desvio padrão.

Umidade das frações (g $100 \mathrm{~g}^{-1}$ ): polpa, 86,5 $\pm 1,58$; casca, $58,7 \pm 1,57$ e sementes, $31,6 \pm 1,29$.

verificaram que a polpa constituiu $63 \%$ do fruto, a casca $25 \%$, as sementes $6 \%$, eixo floral $1,5 \%$ e peso total de $275 \mathrm{~g}$.

\section{Composição centesimal}

As sementes apresentaram os teores mais elevados de proteína bruta e extrato etéreo (EE) quando comparados com a casca (Tabela 2).

Nas sementes, o teor de EE foi de 27,32g $100 \mathrm{~g}^{-1}$ MS (Tabela 2). FREIRE (2001) encontrou 48,6g $100 \mathrm{~g}^{-1} \mathrm{MS}$ de óleo bruto em sementes de mamona, valores estes bastante superiores aos verificados para a atemoia.

Em relação às cinzas, ocorreu diferença significativa entre os tratamentos, sendo que os níveis mais elevados foram encontrados na casca, seguidos pela polpa e sementes. Os teores de fibras alimentares (FA) foram mais elevados nas sementes. A polpa apresentou teores de fibras alimentares solúveis mais altos, quando comparada com as demais frações (Tabela 2).

ROESLER et al. (2007), realizando estudo dos constituintes nutricionais de araticum (Annona crassiflora), encontraram teores de EE de 15,1g $100 \mathrm{~g}^{-1}$, proteína bruta $10,4 \mathrm{~g} 100 \mathrm{~g}^{-1} \mathrm{MS}$, enquanto, para as cinzas, $1,14 \mathrm{~g} 100 \mathrm{~g}^{-1}$ em sementes. Na polpa

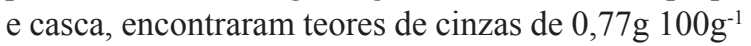

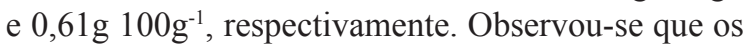
teores desses nutrientes, em qualquer fração estudada, foram inferiores ao da atemoia Gefner.

O extrato não nitrogenado (ENN) constituise principalmente de açúcares. Maiores teores foram encontrados na polpa, seguidos pela casca (Tabela 2). $\mathrm{Na}$ casca, estava aderida uma porção de polpa, sendo, portanto, parte desses açúcares atribuídos à polpa.

Ácido ascórbico, açúcares totais e minerais

Os teores de ácido ascórbico nas frações do fruto mostraram que a casca apresentou maiores teores, com $105,41 \mathrm{mg} 100 \mathrm{~g}^{-1}$. A polpa apresentou

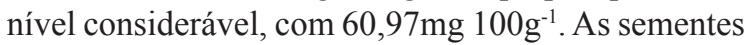
foram muito inferiores à polpa e casca, com $8,18 \mathrm{mg}$ $100 \mathrm{~g}^{-1}$ (Tabela 3). GONÇALVES (2008), em seu trabalho com a polpa de graviola, que é uma fruta do gênero Annona, encontraram $82 \mathrm{mg}^{100 \mathrm{~g}^{-1}}$ de ácido ascórbico.

A ingestão diária recomendada (IDR) de vitamina $\mathrm{C}$ é de $60 \mathrm{mg}$, para um indivíduo adulto (ANVISA, 2004). Portanto, a casca de atemoia representa boa fonte dessa vitamina, podendo ser utilizada nas indústrias de alimentos e de cosméticos. A ingestão de $57 \mathrm{~g}$ de pó de casca de atemoia Gefner representa, em média, $60 \mathrm{mg}$ de ácido ascórbico ingerido, que supriria praticamente a necessidade diária. FETT (2002) afirma que é muito importante consumi-la, pois exerce função antioxidante.

A atemoia apresentou teores de açúcares

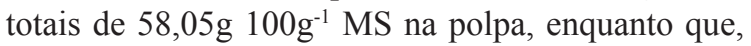
na casca e sementes, os valores foram inferiores

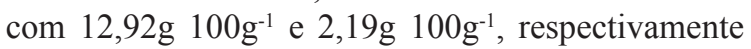
(Tabela 3).

Tabela 2 - Composição centesimal, em g $100 \mathrm{~g}^{-1}$ de matéria seca, das frações de atemoia Gefner.

\begin{tabular}{|c|c|c|c|c|c|c|c|}
\hline \multirow{2}{*}{ Frações do fruto } & \multirow{2}{*}{ Proteína bruta } & \multirow{2}{*}{ Extrato etéreo } & \multirow{2}{*}{ Cinzas } & \multicolumn{3}{|c|}{ 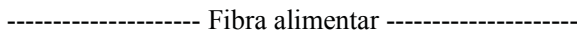 } & \multirow{2}{*}{ ENN } \\
\hline & & & & Insolúvel & Solúvel & Total & \\
\hline Casca & $10,88 \pm 0,42 b$ & $2,80 \pm 0,18 \mathrm{~b}$ & $5,64 \pm 0,25 \mathrm{a}$ & $52,82 \pm 1,43 b$ & $\mathrm{ND}^{* *}$ & $52,83 \pm 1,42 b$ & $27,85 \mathrm{~b}$ \\
\hline Polpa & $6,84 \pm 0,43 \mathrm{c}$ & $1,51 \pm 0,25 \mathrm{c}$ & $4,74 \pm 0,31 \mathrm{~b}$ & $20,56 \pm 1,27 \mathrm{a}$ & $2,48 \pm 0,51$ & $23,04 \pm 1,03 \mathrm{c}$ & $63,91^{\mathrm{a}}$ \\
\hline Sementes & $14,79 \pm 0,46 \mathrm{a}$ & $27,32 \pm 1,03 \mathrm{a}$ & $1,73 \pm 0,06 \mathrm{c}$ & $56,00 \pm 2,38 \mathrm{c}$ & $1,53 \pm 0,46$ & $57,53 \pm 2,38 \mathrm{a}$ & $0,16 \mathrm{c}$ \\
\hline
\end{tabular}

Os dados são a média de sete repetições.

Letras diferentes indicam diferença significativa, pelo teste de Tukey, com probabilidade de 5\%.

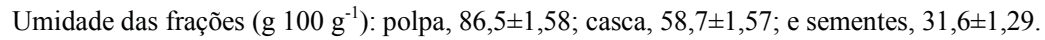

*ENN: Extrato não nitrogenado.

**ND: Não detectado.

Ciência Rural, v.43, n.12, dez, 2013. 
Tabela 3 - Teores de ácido ascórbico (mg $100 \mathrm{~g}^{-1} \mathrm{MS}$ ), açúcares totais (g $100 \mathrm{~g}^{-1} \mathrm{MS}$ ), macrominerais P, K, Ca, Mg e S (g $\left.100 \mathrm{~g}{ }^{-1} \mathrm{MS}\right)$ e microminerais $\mathrm{Cu}, \mathrm{Mn}, \mathrm{Zn}$ e Fe ( $\left.\mathrm{mg} \mathrm{kg}^{-1} \mathrm{MS}\right)$ nas frações de atemoia 'Gefner'.

\begin{tabular}{|c|c|c|c|c|c|c|c|c|c|c|c|}
\hline \multirow[b]{2}{*}{$\begin{array}{l}\text { Frações do } \\
\text { fruto }\end{array}$} & \multirow[b]{2}{*}{$\begin{array}{c}\text { Ácido } \\
\text { ascórbico }\end{array}$} & \multirow[b]{2}{*}{$\begin{array}{c}\text { Açúcares } \\
\text { totais }\end{array}$} & \multicolumn{5}{|c|}{ 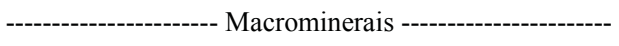 } & \multicolumn{4}{|c|}{ 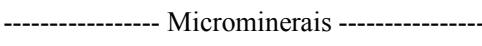 } \\
\hline & & & $\mathrm{P}$ & $\mathrm{K}$ & $\mathrm{Ca}$ & $\mathrm{Mg}$ & $\mathrm{S}$ & $\mathrm{Cu}$ & $\mathrm{Mn}$ & $\mathrm{Zn}$ & $\mathrm{Fe}$ \\
\hline Casca & $\begin{array}{c}105,41 \\
\pm 2,56 \mathrm{a}\end{array}$ & $\begin{array}{c}12,92 \\
\pm 0,68 \mathrm{~b}\end{array}$ & $\begin{array}{c}0,19 \\
\pm 0,00 \mathrm{~b}\end{array}$ & $\begin{array}{c}1,66 \\
\pm 0,09 b\end{array}$ & $\begin{array}{c}0,14 \\
\pm 0,01 \mathrm{~b}\end{array}$ & $\begin{array}{c}0,12 \\
\pm 0,01 \mathrm{a}\end{array}$ & $\begin{array}{c}0,17 \\
\pm 0,01 \mathrm{a}\end{array}$ & $\begin{array}{c}10,78 \\
\pm 0,37 \mathrm{~b}\end{array}$ & $\begin{array}{c}7,20 \\
\pm 0,68 \mathrm{~b}\end{array}$ & $\begin{array}{c}11,60 \\
\pm 0,88 \mathrm{~b}\end{array}$ & $\begin{array}{c}23,96 \\
\pm 0,90 \mathrm{~b}\end{array}$ \\
\hline Polpa & $\begin{array}{r}60,97 \\
\pm 1,66 \mathrm{~b}\end{array}$ & $\begin{array}{c}58,05 \\
\pm 2,75 \mathrm{a}\end{array}$ & $\begin{array}{c}0,15 \\
\pm 0,02 \mathrm{c}\end{array}$ & $\begin{array}{c}1,70 \\
\pm 0,16 \mathrm{a}\end{array}$ & $\begin{array}{c}0,13 \\
\pm 0,01 \mathrm{c}\end{array}$ & $\begin{array}{c}0,12 \\
\pm 0,01 \mathrm{a}\end{array}$ & $\begin{array}{c}0,16 \\
\pm 0,02 \mathrm{a}\end{array}$ & $\begin{array}{c}5,06 \\
\pm 0,53 \mathrm{c}\end{array}$ & $\begin{array}{c}5,84 \\
\pm 0,36 \mathrm{c}\end{array}$ & $\begin{array}{c}6,60 \\
\pm 1,03 \mathrm{c}\end{array}$ & $\begin{array}{c}21,89 \\
\pm 0,59 \mathrm{c}\end{array}$ \\
\hline Sementes & $\begin{array}{c}8,18 \\
\pm 0,70 \mathrm{c}\end{array}$ & $\begin{array}{c}2,19 \\
\pm 0,07 \mathrm{c}\end{array}$ & $\begin{array}{c}0,21 \\
\pm 0,01 \mathrm{a}\end{array}$ & $\begin{array}{c}0,50 \\
\pm 0,03 \mathrm{c}\end{array}$ & $\begin{array}{c}0,18 \\
\pm 0,01 \mathrm{a}\end{array}$ & $\begin{array}{c}0,12 \\
\pm 0,01 \mathrm{a}\end{array}$ & $\begin{array}{c}0,16 \\
\pm 0,03 \mathrm{a}\end{array}$ & $\begin{array}{c}19,95 \\
\pm 1,26 \mathrm{a}\end{array}$ & $\begin{array}{c}13,65 \\
\pm 0,99 \mathrm{a}\end{array}$ & $\begin{array}{c}36,70 \\
\pm 1,85 \mathrm{a}\end{array}$ & $\begin{array}{c}36,93 \\
\pm 1,55 \mathrm{a}\end{array}$ \\
\hline
\end{tabular}

Os dados são a média de sete repetições \pm desvio padrão.

Letras diferentes indicam diferença significativa pelo teste de Tukey, a 5\% de probabilidade.

ROESLER et al. (2007), realizando estudo dos constituintes químicos nas frações do fruto araticum, gênero Annona, encontraram teores de açúcares totais de $19,05 \mathrm{~g} 100 \mathrm{~g}^{-1}$ na polpa, $19,23 \mathrm{~g}$ $100 \mathrm{~g}^{-1}$ na casca, e $20,14 \mathrm{~g} 100 \mathrm{~g}^{-1}$ MS nas sementes, enquanto que AGOSTINI et al. (1995) encontraram

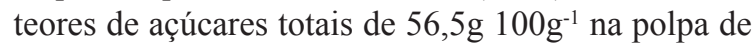
marolo (Annona coriaceae).

Observou-se neste trabalho que a casca de atemoia apresentou teor elevado de potássio (1.660mg $\left.100 \mathrm{~g}^{-1} \mathrm{MS}\right)$ e baixos teores de cálcio (140mg 100 g $\left.\mathrm{g}^{-1} \mathrm{MS}\right)$ e magnésio (120mg $100 \mathrm{~g}^{-1}$ MS) (Tabela 3). GONDIM et al. (2005), analisando minerais em cascas de abacate, abacaxi, banana, mamão, maracujá, melão e tangerina mostrou que as cascas de tangerina são boas fontes de minerais, com maiores concentrações de potássio $(1.175,5 \mathrm{mg}$ $100 \mathrm{~g}^{-1} \mathrm{MS}$ ), seguido do cálcio (941mg $\left.100 \mathrm{~g}^{-1} \mathrm{MS}\right) \mathrm{e}$

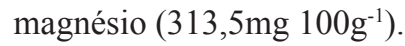

FELIPE et al. (2006) encontraram os seguintes teores (mg $100 \mathrm{~g}^{-1} \mathrm{MS}$ ) na casca de manga e de maracujá, respectivamente: potássio: 205,7 e 690,02; cálcio: 39,80 e 58,65; cobre: 0,41 e 1,41; zinco: 13,49 e 1,82; manganês: 0,73 e 1,26 e ferro 3,93 e 13,52 .

Os teores de magnésio e enxofre encontrados para essa fruta foram considerados baixos e não houve diferença significativa entre as três frações, com teor médio de $0,12 \mathrm{~g} 100 \mathrm{~g}^{-1} \mathrm{MS}$ e $0,16 \mathrm{~g} 100 \mathrm{~g}^{-1} \mathrm{MS}$, respectivamente. As sementes apresentaram os maiores teores em todos os microminerais analisados: cobre, manganês, zinco e ferro e a polpa os menores (Tabela 3).
O cobre possui papel importante na ativação enzimática. Os teores de cobre encontrados em atemoia Gefner foram de $19,9 \mathrm{mg} \mathrm{kg}^{-1}$ na semente, $10,78 \mathrm{mg} \mathrm{kg}^{-1}$ na casca e $5 \mathrm{mg} \mathrm{kg}^{-1}$ na polpa. Os teores de zinco foram altos na semente $\left(36,7 \mathrm{mg} \mathrm{kg}^{-1}\right)$, seguido da casca $\left(11,6 \mathrm{mg} \mathrm{kg}^{-1}\right)$ e polpa $\left(6,6 \mathrm{mg} \mathrm{kg}^{-1}\right)$, sendo estatisticamente diferentes (Tabela 3 ).

Os teores de ferro apresentaram diferença significativa em todas as frações estudadas, com valores de $36,9 \mathrm{mg} \mathrm{kg}^{-1}$ na semente, $23,9 \mathrm{mg} \mathrm{kg}^{-1}$ na casca e $21,9 \mathrm{mg} \mathrm{kg}^{-1}$ na polpa. A IDR de ferro é de $14 \mathrm{mg}$ e de zinco é de $15 \mathrm{mg}$ (ANVISA, 2004). A ingestão de $100 \mathrm{~g}$ de pó de casca e semente de atemoia Gefner, respectivamente, representa $2,40 \mathrm{mg}$ e $3,69 \mathrm{mg}$ de ferro, zinco de $1,16 \mathrm{mg}$ e $3,67 \mathrm{mg}$, sendo essas quantidades consideradas baixas e não suprindo as necessidades diárias (Tabela 3).

\section{Compostos bioativos}

A casca apresentou maiores teores de inibidores de tripsina do que a polpa, $176,67 \mu \mathrm{mol} \mathrm{min}^{-1}$ $\mathrm{g}^{-1} \mathrm{MS}$ e $95,16 \mu \mathrm{mol} \mathrm{min}^{-1} \mathrm{~g}^{-1} \mathrm{MS}$, respectivamente (Tabela 4). Os compostos fenólicos estavam em maior quantidade na casca do que nas outras frações, com 94,19mg $100 \mathrm{~g}^{-1} \mathrm{MS}$, seguido das sementes, com 53,02mg $100 \mathrm{~g}^{-1} \mathrm{MS}$ e polpa, $10,38 \mathrm{mg} 100 \mathrm{~g}^{-1}$ MS. ROESLER et al. (2007), avaliando o fruto de araticum (Annona crassiflora), encontraram níveis de compostos fenólicos nas sementes de $136,99 \mathrm{~g} \mathrm{~kg}^{-1}$ MS, na casca de $90,72 \mathrm{~g} \mathrm{~kg}^{-1} \mathrm{MS}$ e na polpa de $20,31 \mathrm{~g}$ $\mathrm{kg}^{-1} \mathrm{MS}$, valores estes superiores aos encontrados em atemoia. 
Tabela 4 - Teores de inibidores de tripsina e compostos fenólicos (em matéria seca) e atividade hemaglutinante nas frações de atemoia Gefner.

\begin{tabular}{|c|c|c|c|}
\hline Frações do fruto & Inibidores tripsina $\left(\mu \mathrm{mol} \min ^{-1} \mathrm{~g}^{-1} \mathrm{MS}\right)$ & Compostos fenólicos (mg $100 \mathrm{~g}^{-1} \mathrm{MS}$ ) & Lectinas (UH mg proteína $\left.^{-1}\right)^{*}$ \\
\hline Casca & $176,67 \pm 2,22$ & $94,19 \pm 5,25 \mathrm{a}$ & $2.617,29 \pm 34,14$ \\
\hline Polpa & $95,16 \pm 19,15$ & $10,38 \pm 0,46 \mathrm{c}$ & $722,35 \pm 78,13$ \\
\hline Sementes & $\mathrm{ND}^{* *}$ & $53,02 \pm 3,69 b$ & ND** \\
\hline
\end{tabular}

Os dados são a média de sete repetições \pm desvio padrão.

Letras diferentes indicam diferença significativa pelo teste de Tukey, a 5\% de probabilidade.

*O valor expressa o inverso do título da maior diluição na base 2 , que ainda produziu aglutinação visível em número de unidades hemaglutinantes $(\mathrm{UH})$, em cada $100 \mu \mathrm{L}$ de amostra utilizados no ensaio com sangue humano tipo $\mathrm{A} \mathrm{Rh+}$.

**ND: Não detectado.

Não foi verificada atividade hemaglutinante na semente do fruto atemoia, entretanto a casca apresentou 2.617,29UH mg proteína ${ }^{-1}$ MS e a polpa 722,35UH mg proteína-1 MS (Tabela 4).

\section{CONCLUSÃO}

Com os resultados apresentados no presente trabalho, as duas frações, casca e sementes, poderão ser aproveitadas na alimentação, agregando maior valor ao fruto, pois representam, aproximadamente, $40 \%$ do total do fruto. As análises químicas evidenciaram que a casca e as sementes da atemoia apresentaram, em geral, teores de nutrientes maiores, quando comparadas com a parte comestível, e o teor de compostos bioativos é relativamente baixo e não causaria malefícios à saúde do organismo.

\section{AGRADECIMENTO}

Agradecemos à Fundação de Amparo à Pesquisa do estado de Minas Gerais (FAPEMIG) pela bolsa de Mestrado e apoio financeiro.

\section{REFERÊNCIAS}

AGENCIA NACIONAL DE VIGILÂNCIA SANITÁRIA. Ingestão diária recomendada (IDR) para proteínas, vitaminas e minerais, 2004. Disponível em: <http://www.anvisa.gov.br/ base/visadoc/CP/CP[8989]pdf>. Acesso em: 7 fev. 2011.

AGOSTINI, T. et al. Chemical characterization of the oil and pulp of marolo (Annona coriaceae). Archivos Latinoamericanos de Nutrición, Caracas, v.45, n.3, p.237-41, Sept, 1995.

ASSOCIATION OF OFFICIAL ANALYTICALCHEMISTS. Official methods of analysis of Association of Official Analytical Chemists. 18.ed. Maryland, 2005. 1094p.

CALDERÓN DE LA BARCA, A.M. et al. Effect of extraction of a hemaglutinin on the nutritive value of Amaranthus leucocarpus seeds. Journal of Food Science, Chicago, v.50, p.1770- 1772, 1985.
CAXITO, A.M. Atemoia do Jaíba/MG seduz a Europa. Portal Abanorte. 2009. Disponivel em: <http://www.abanorte.com. br/noticias/noticias-principal/atemoia-do-jaiba-mg-seduz-aeuropa/>. Acesso em: 9 maio, 2009.

DISCHE, Z. General color reactions. In: WHISTLER, R.L.; WOLFRAM, M.L. (Ed.). Carboidrate chemistry. New York: Academic, 1962. p.477-512.

FELIPE, E.M. de F. et al. Avaliação da qualidade de parâmetros minerais de pós-alimentícios obtidos de casca de manga e maracujá. Alimentos e Nutrição, Araraquara v.17, n.1, p.79-83, 2006.

FETT, C. Ciência da suplementação alimentar. 2.ed. Rio de Janeiro: Sprint, 2002. 390p.

FREIRE, R.M.M. Ricinoquímica. In: AZEVEDO, D.M.P.; LIMA, E.F. O agronegócio da mamona no Brasil. Campina Grande: Embrapa Algodão; Brasília: Embrapa Informação Tecnológica, 2001. p.295-335.

GONDIM, J.A.M. et al. Composição centesimal e de minerais em cascas de frutas. Ciência e Tecnologia de Alimentos, Campinas, v. 25, n. 4 , p.825-827, 2005.

GONÇALVES, A.E. de S.S. Avaliação da capacidade antioxidante de frutas e polpas de frutas nativas e determinação dos teores de flavonóides e vitamina C. 2008. 88f. Dissertação (Mestrado em Bromatologia) - Curso de Pós-graduação em Ciência dos Alimentos, Universidade de São Paulo, SP.

KAKADE, M.L. et al. The evaluation of natural vs. Synthetic substrates for measuring the antitryptic activity of soybean sample. Cereal Chemistry, St. Paul, v.46, p.518-526, 1969.

MALAVOLTA, E. et al. Avaliação do estado nutricional das plantas: princípios e aplicações. Piracicaba: POTAFÓS, 1997. 201 p.

NEVES, C.S.V.J.; YUHARA, E.N. Caracterização dos frutos de cultivares de atemóia produzidos no norte do Paraná. Semina: Ciências Agrárias, Londrina, v.24, n.2, p.311-314, 2003.

ROESLER. R. et al. Atividade antioxidante de frutas do cerrado. Ciência e Tecnologia Alimentos, Campinas, v.27, n.1, p.53-60, 2007.

STROHECKER, R.; HENNING, H.M. Análises de vitaminas: metodos comprobados. Madrid: Paz Montalvo, 1967. 428p. 